\begin{tabular}{|c|c|c|c|c|}
\hline & Epoq1 & & $\varphi-m$ & $\mathrm{ob}$ \\
\hline $89 \mathrm{I}$ & Juillet & I 6 & $0^{\circ} 18^{\prime} 57^{\prime \prime} \cdot 51$ & 6 \\
\hline & $》$ & 20 & 57.53 & \\
\hline & 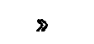 & 26 & 57.66 & \\
\hline & Août & I 4 & 57.69 & \\
\hline & 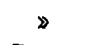 & 28 & 57.74 & \\
\hline & Sept. & I I & 57.77 & \\
\hline & $\triangleright$ & I 7 & 57.86 & \\
\hline & 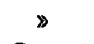 & 28 & 57.91 & \\
\hline & Oct. & 3 & 57.85 & \\
\hline & 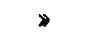 & 6 & 57.92 & \\
\hline & 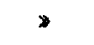 & I I & 57.90 & \\
\hline & $\triangleright$ & I 5 & 57.83 & \\
\hline & 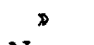 & 30 & 57.75 & 7 \\
\hline & Nov. & I 2 & 57.74 & I I \\
\hline & 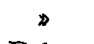 & 28 & 57.75 & \\
\hline & Déc. & 22 & 57.69 & \\
\hline 892 & Janv. & ro & 57.63 & \\
\hline & Févr. & $\mathbf{I}$ & 57.55 & \\
\hline & $\triangleright$ & 23 & 57.53 & \\
\hline & Mars & I & 57.49 & \\
\hline & > & 19 & 57.54 & \\
\hline & Avril & 4 & 57.52 & \\
\hline & . & 24 & 57.47 & \\
\hline & Mai & 8 & 57.49 & \\
\hline & $\gg$ & I I & 57.37 & \\
\hline & D. & 23 & 57.31 & \\
\hline
\end{tabular}

Ces valeurs $\varphi-m$ sont portées sur la planche et avec les points reçus on a tracé la courbe moyenne; on

Poulkovo 1892 Juin 3 . peut estimer de 0."03-0"04 l'erreur probable de chacun de ces points. A l'aide de cette courbe nous avons trouvé l'époque de maximum de la latitude en 1891 près de 4 Octobre avec la valeur $\varphi-m=0^{\circ} 18^{\circ} 57^{\prime \prime} 88$. Par rapport à l'époque de minimum en 1892 on peut dire seulement, que probablement elle n'aura pas lieu avant la fin du mois de Mai; les grandes fluctuations de la courbe à cette époque s'expliquent par les mauvaises images des étoiles. En comparant notre courbe avec la courbe définitive pour I 890-189I, déduite par M. Wanach (A.N. 3092) et admettant pour $m$ la valeur $59^{\circ} 27^{\prime} 20^{\prime \prime} 56$ qui suit des déclinaisons donneés par lui, nous trouvons les résultats suivantes :

Maximum de la lat. en 1890 Sept. i $4 \varphi=59^{\circ} 46^{\prime}$ I $8^{\prime \prime} 39$ Minimum $\gg \gg$ 1891 Avril I 5 I 7.79 Maximum 》 \ $\gg 1891$ Oct. $4 \quad 18.44$ Minimum » » $₫ 892$ la fin du mois de Mai.

De la comparaison des époques des maxima on obtient pour la période du phénomène presque $\mathrm{I}_{3}$ mois ( 386 jours); cela se confirme aussi pour les époques des minima. Ayant en vue les erreurs dans la détermination des époques maxima et minima, on peut dire, il me semble, que les observations de notre observatoire confirment la période de 427 jours, indiquée par $M$. Chandler (Astronomical Journal Nr. 248-251); mais cette question peut être résolue définitivement à l'aide d'une série des observations plus longue.

\title{
Elemente und Ephemeride des Planeten (288) Glauke.
}

Da eine Ephemeride für $\delta$ III (288) Glauke nicht veröffentlicht ist, leitete ich aus den Beobachtungen 1890 und 1891 unter Berücksichtigung der Jupiterstörungen folgende Elemente II (288) Glauke ab :
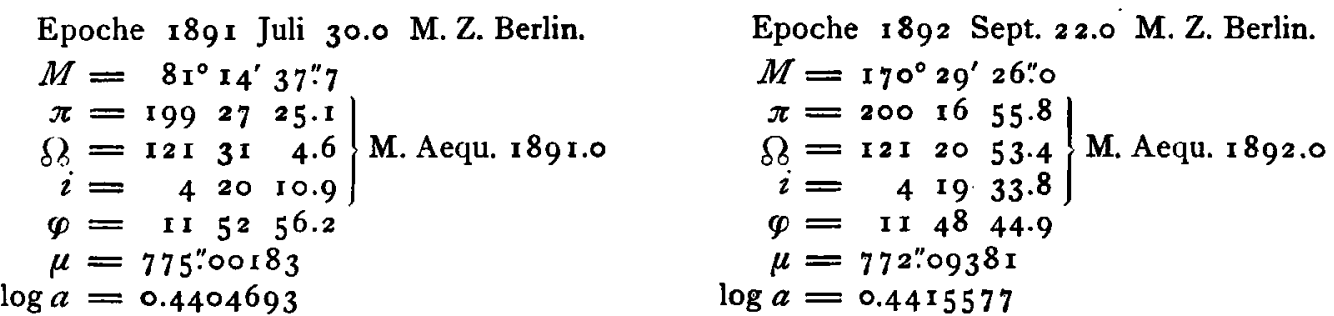

Die Vergleichung der Elemente $189 \mathrm{r}$ mit den drei Beobachtungen 1891 ergab im Mittel aus 2 Nizza und I Wien die Correction der neuen Ephemeride $189 x+0.53$ +.1." 2.

Mit den Elementen 1892 berechnete ich für 8 III die folgende

Ephemeride für $12^{\text {h }}$ M. Z. Berlin.

\begin{tabular}{|c|c|c|c|c|}
\hline 1892 & $\alpha$ app. & $\delta$ app. & $\log \Delta$ & Ab. Zt. \\
\hline 14 & $I^{\mathrm{h}} 26^{\mathrm{m}} 17^{\mathrm{s}}$ & $+2^{\circ} 57^{\prime} \cdot 9$ & $0.3^{81} 6$ & $9^{m} 5^{8}$ \\
\hline 15 & 2542 & 5 & $3^{8 c}$ & 55 \\
\hline 16 & 256 & 248 & 37 & $5^{2}$ \\
\hline I 7 & 2429 & 243 & & 49 \\
\hline 18 & $235 I$ & 238 & $3 ?$ & 47 \\
\hline I 9 & I 2312 & +233.2 & 0.3764 & 1944 \\
\hline
\end{tabular}

\begin{tabular}{|c|c|c|c|c|}
\hline 1892 & $\alpha$ app. & $\delta$ app. & $\log A$ & $\mathrm{Ab} . \mathrm{Zt}$ \\
\hline pt. 20 & $1^{\mathrm{h}} 22^{\mathrm{m}} 33^{\mathrm{s}}$ & $+2^{\circ} 28: \mathrm{I}$ & .3755 & $19^{\mathrm{m}} 4 \mathrm{I}^{\mathrm{s}}$ \\
\hline 21 & 2152 & 223.0 & 3 & 39 \\
\hline 22 & 2 III & $\begin{array}{l}2 \quad 17.8\end{array}$ & $373^{8}$ & 3 \\
\hline 23 & 2030 & 212.6 & 37 & 3 \\
\hline 24 & 1947 & $7 \cdot 4$ & 3723 & 3 \\
\hline 25 & 19 & +2 & 3717 & 19 \\
\hline
\end{tabular}




\begin{tabular}{|c|c|c|c|c|}
\hline 1892 & $\alpha$ app. & $\delta$ app. & $\log \Delta$ & Ab. Zt. \\
\hline Sept. 26 & $1^{h} 18^{m} 20^{s}$ & $+I^{\circ} 5^{6: 8}$ & $0.37 \times 1$ & $19^{\mathrm{m}} 30^{\mathrm{s}}$ \\
\hline 27 & $173^{6}$ & I 51.5 & $37 \circ 5$ & 28 \\
\hline 28 & 1651 & $I: 46.2$ & 3700 & 27 \\
\hline 29 & 166 & I 40.9 & 3696 & 25 \\
\hline 30 & 1520 & I. 35.6 & 3692 & 24 \\
\hline Oct. I & 1434 & I 30.2 & 3688 & 23 \\
\hline 2 & 1348 & I 24.9 & $3^{685}$ & 23 \\
\hline 3 & 131 & 119.6 & $3^{68} 3$ & 22 \\
\hline 4 & I2 I 4 & I 14.3 & $368 I$ & 22 \\
\hline 5 & I I 27 & I $9 . \mathrm{I}$ & 3680 & 21 \\
\hline 6 & IO 40 & I $\quad 3.9$ & 3679 & 2 I \\
\hline 7 & 952 & o $\quad 58.7$ & $3^{679}$ & 21 \\
\hline$\gamma$ & 95 & - 53.5 & 3680 & 21 \\
\hline 9 & $8 I 7$ & 048.4 & 3681 & 22 \\
\hline 810 & 729 & - 43.3 & 3683 & 22 \\
\hline $1 \mathrm{I}$ & $64 I$ & - $\quad 3^{8.3}$ & $3^{685}$ & 23 \\
\hline I 2 & I $\quad 5 \quad 54$ & to 33.3 & $0.3^{687}$ & 1923 \\
\hline
\end{tabular}

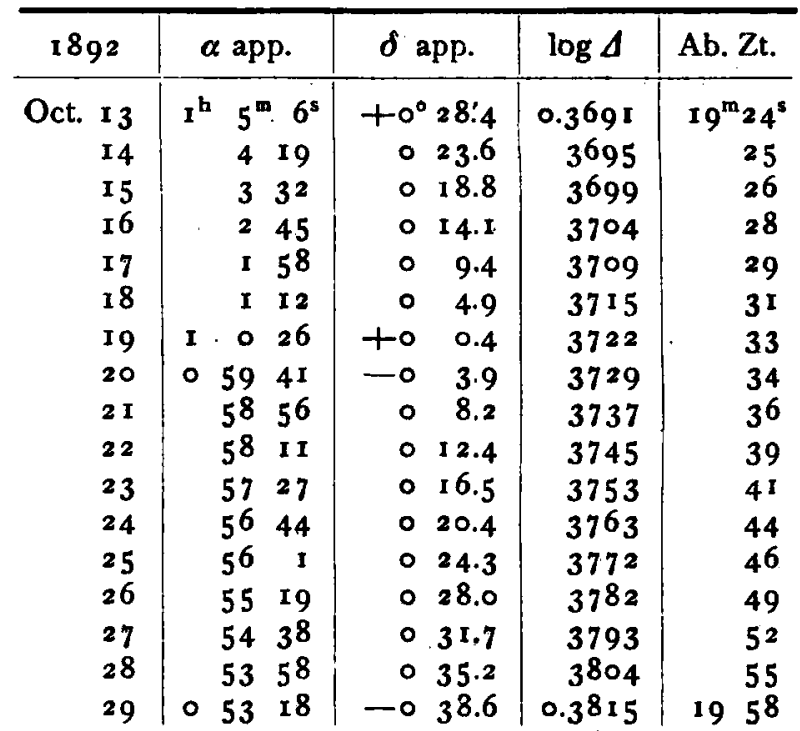

Obgleich zu hoffen ist, dass die Correction der Ephemeride klein sein werde, ewähne ich doch, dass für Sept. I 7 einer Aenderung $\Delta \alpha+1^{\mathrm{m}} \Delta d+5: 85$ entsprechen würde.

Von Sept. 20 bis Oct. 18 wird Peters Karte 4 nützlich sein. Der Planet ist 13. Grösse.

Dusseldorfer Sternwarte 1892 August $3 \mathrm{r}$.

Robert Luther.

Beobachtungen des Cometen 1892 ... (Brooks Aug. 27).

\begin{tabular}{|c|c|c|c|c|c|c|c|c|c|c|}
\hline 1892 & M. Ortszeit & $\Delta \alpha$ & $\Delta \delta$ & Vgl. & $\alpha$ app. & $\log p .4$ & $\delta$ app. & $|\log p \cdot \Delta|$ & Red.ad 1. app. & * \\
\hline
\end{tabular}

Am Refractor von $20 \mathrm{~cm}$ Oeffnung der Sternwarte in Jena von Dr. O. Knopf.

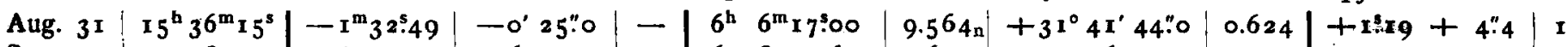

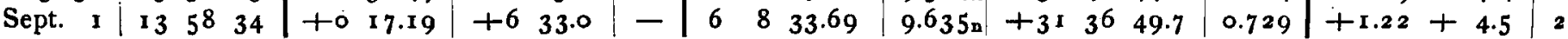

Die Beobachtungen sind vermittelst eines Kreismikrometers mit hellen Linien im dunklen Feld angestellt worden.

Au Grand Equatorial de l'Observatoire de Bordeaux par M. G. Rayet.

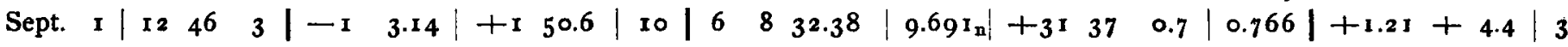
A l'Equatorial Brunner (omi6) de l'Observatoire de Lyon par M. G. Le Cadet.

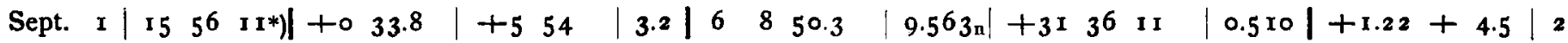
La comète est parfois voilée par des nuages légers.

*) T. m. Paris. Auf der Sternwarte in Kremsmünster von Prof. F. Schwab.

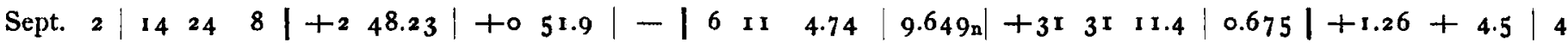
Auf der Sternwarte in Kopenhagen von Herrn C. F. Pechüle.

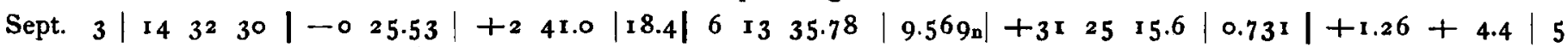
Mittlere Oerter der Vergleichsterne für 1892.0 .

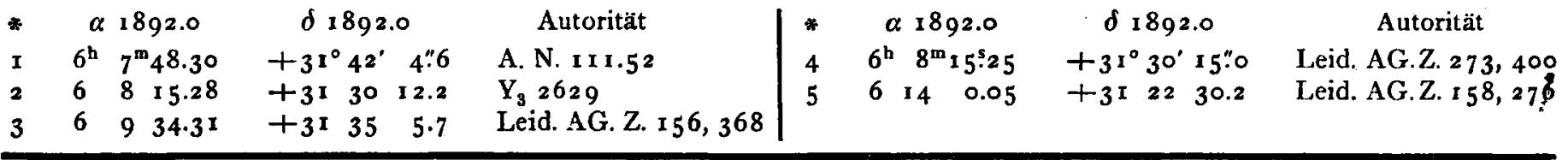

Inhalt:

Zu Nr. 3II2. E.v. Haerdtl. Ephemeride fiir den periodischen Cometen Winnecke $1892 \ldots 24$ r. - B. Wanach. Ableitung der Polhöhenschwankungen aus älteren Pulkowaer Beobachtungen im ersten Vertical. 245. - S. Kostinsky. Sur les variations de la latitude de Poulkovo en 1891-1892. 251. $-R$. Luther. Elemente und Ephemeride des Planeten (288) Glauke. 253. - Beobachtungen des Cometen $1892 \ldots$ (Brooks Aug. 27). 255. 